\title{
Combination of Treadmill Aerobic Exercise with Bifidobacterium longum OLP-01 Supplementation for Treatment of High-Fat Diet-Induced Obese Murine Model
}

\author{
Yi-Ju Hsu $^{a}$ Chien-Chao Chiu ${ }^{a}$ Mon-Chien Lee ${ }^{a}$ Wen-Ching Huang ${ }^{b}$ \\ ${ }^{a}$ Graduate Institute of Sports Science, National Taiwan Sport University, Taoyuan, Taiwan; ${ }^{b}$ Department of Exercise \\ and Health Science, National Taipei University of Nursing and Health Sciences, Taipei, Taiwan
}

\section{Keywords}

Obesity · Probiotics · Exercise training · Oxidative stress .

Hyperglycemia

\begin{abstract}
Introduction: Obesity, which can result from disease, genetics, nutrition, lifestyle, and insufficient physical activity, substantially increases an individual's risk of complications and comorbidities. Exercise can be an effective strategy for achieving an energy balance and physiological fitness as part of obesity management. Additionally, probiotics, which are isolated from food and the environment, are being rapidly developed and have functional benefits for mitigating various metabolic dysfunctions associated with obesity. The potentially positive physiological and functional effects of exercise, probiotics, and exercise combined with probiotics should be elucidated in a model of diet-induced obesity. Methods: Bifidobacterium longum subsp. longum OLP-01 (OLP-01) was isolated from an elite Olympic-level athlete who exhibited physiological adaptations to peripheral fatigue caused by exercise training. In this current study, ICR strain mice were fed a high-fat diet (HFD) for 4 weeks to rep-
\end{abstract}

karger@karger.com www.karger.com/ofa

Karger $\stackrel{\text { ' }}{5}$

BOPEN ACCESS
(C) 2021 The Author(s)

Published by S. Karger AG, Basel

This is an Open Access article licensed under the Creative Commons Attribution-NonCommercial-4.0 International License (CC BY-NC) (http://www.karger.com/Services/OpenAccessLicense), applicable to the online version of the article only. Usage and distribution for commercial purposes requires written permission. licate an obesity model. The mice were divided into 5 groups according to the diet administered: control with normal diet, only HFD, HFD + exercise, HFD + OLP, and HFD + exercise + OLP groups. They were administered the probiotic and/or treadmill exercise training for 5 weeks, and their growth curve, physical activity, physiological adaptation, biochemical parameters, body composition, and glucose tolerance were assessed. Results: Compared with only exercise or only probiotics, a combination of probiotics and exercise significantly improved the weight, glucose tolerance, fat composition, and exercise-related oxidative stress of mice. Regular and programmed exercise with sufficient rest may be crucial to obesity improvement, and a combination of probiotics and exercise may synergistically assist obesity management and health promotion. Conclusion: OLP-01 probiotics combined with exercise training can be employed as a strategy for treating obesity. However, the exact regulatory mechanisms underlying this effect, possibly involving microbiota and associated metabolites, warrant further investigation.

(c) 2021 The Author(s)

Published by S. Karger AG, Basel

Y.-J.H., C.-C.C., and M.-C.L. contributed equally to this study.
Correspondence to:

Wen-Ching Huang, wenching@ntunhs.edu.tw 


\section{Introduction}

The incidence of overweight and obesity is increasing at an alarming rate, not only in adults of working age but also children and older adults. Overweight and obesity are associated with higher risks of complications such as diabetes, cardiovascular disease, hypoventilation, chronic kidney disease, steatohepatitis, subfertility, gastroesophageal reflux, cancer, pharmacokinetic alterations, impaired physical functions, poor self-perceived health, and negative body image, and they incur an economic burden [1]. Indexes such as body mass index (BMI), waist circumference, and waist-to-hip ratio have been widely applied to evaluate obesity [2]. The World Health Organization reported that, in 2016, 39\% of the adults worldwide were overweight and nearly $13 \%$ were obese. According to the Health Promotion Administration of the Ministry of Health and Welfare in Taiwan, the number of overweight individuals (BMI $>25 \mathrm{~kg} / \mathrm{m}^{2}$ ) drastically increased from $32.7 \%$ during $1993-1996$ to $45.4 \%$ during 2013-2016, rendering the Taiwanese population the most overweight in Asia [3]. Another study reported that dietary factors (pattern and food quality), genetics, physical activity, sleep quality, environmental exposure, and related lifestyle interactions can predispose a person to various obesity risk factors [4]. Therefore, animal models were established using high-fat and/or high-density foods, which are the main contributors to obesity, to simulate diet-induced obesity for further investigation of the physiological mechanisms underlying the development of obesity and to develop effective intervention strategies for preventing and managing obesity [5].

Obesity can be controlled by regulating a person's energy balance through proper dietary and exercise interventions. Several types of physical activities and exercise interventions have been suggested for weight and obesity management. However, the different volumes, intensities, and types of exercise can yield different weight loss results. Aerobic exercise alone can contribute to clinically significant weight loss in both men and women [6]. A previous study also demonstrated that aerobic exercise more efficiently reduced body mass and fat mass than resistance exercise did; however, compared with aerobic exercise, resistance exercise considerably increased lean body mass, which is important for middle-aged individuals with overweight and obesity [7]. In addition to aerobic exercise, high-intensity intermittent exercise can more effectively improve muscular adaptation to, in turn, improve fat oxidation, glucose homeostasis, and body composition relative to regular exercise [8]. However, several

Exercise and Probiotics for Diet-Induced Obesity factors such as sex; BMI; the type, intensity, and duration of exercise; dietary habits; and diseases can affect how much weight is lost due to the exercise intervention. In addition to weight loss, the physiological benefits of exercise are also an important factor for health promotion [9].

In addition to the effects of exercise on obesity, dietary and nutritional strategies for promoting weight loss have also been evaluated. Several diets based on restricted caloric intake, for example, intermittent fasting, the juicing diet, and the paleo diet - can cause temporary weight loss; however, individuals tend to regain the lost weight once they resume their normal diet [10]. By contrast, the ketogenic diet has been validated with respect to its abilities to not only treat various diseases but also improve hunger control, fat oxidative metabolism, and body weight management [11]. Probiotics for human use were widely developed and isolated from the intestines of humans and animal, various foods, and environments [12]. They were also investigated for their physiological benefits to immunomodulation and intestinal microbiota composition as well as for their metabolic and therapeutic effects [13]. Other subspecies of Bifidobacterium longum probiotics also reportedly have various health promotion effects. One study discovered that obesity is associated with a reduced amount of the beneficial Bifidobacteria and that oligofructose, a prebiotic, ameliorated obesity-associated inflammation through the critical commensal microflora B. pseudolongum [14]. Obesity-associated pathological characteristics, such as colitis, endotoxemia, and liver steatosis, were alleviated by $B$. longum LC67 supplementation because of suppression of NF- $\kappa \mathrm{B}$ activation and increased AMPK activation and tight junction expression [15]. In addition to affecting obesity, B. longum NCC3001 altered brain activation patterns through reduction of limbic reactivity to negative emotional stimuli and improved depression scores and quality of life in patients with irritable bowel syndrome [16]. In a comparative genome analysis of individuals aged between 0 and 98 years, numerous $B$. longum strains appeared to have been transmittable across families, and certain characteristics, such as carbohydrate metabolism, appeared to have been more prevalent among strains corresponding to specific host ages. Therefore, B. longum strongly affects physiological activities across the human lifespan [17]. Although many studies have focused on the potential benefits of probiotics on obesity, the exact dose of the probiotics and the requisite duration of treatment warrant further investigation [18]. Probiotics can exert functional effects on physiological functional activities as well as weight management. 
Fig. 1. Experimental designs for the effects of exercise and probiotics on a high fat diet (HFD)-induced obesity model. The animals were randomly assigned to the indicated 5 groups (Control, HFD, HFD + OLP-01, HFD + Ex, and HFD + Ex + OLP01). Obesity was induced by an HFD for the whole experimental period, including the induction and experimental phases. Physical fitness and related assessments were performed within the experimental period.

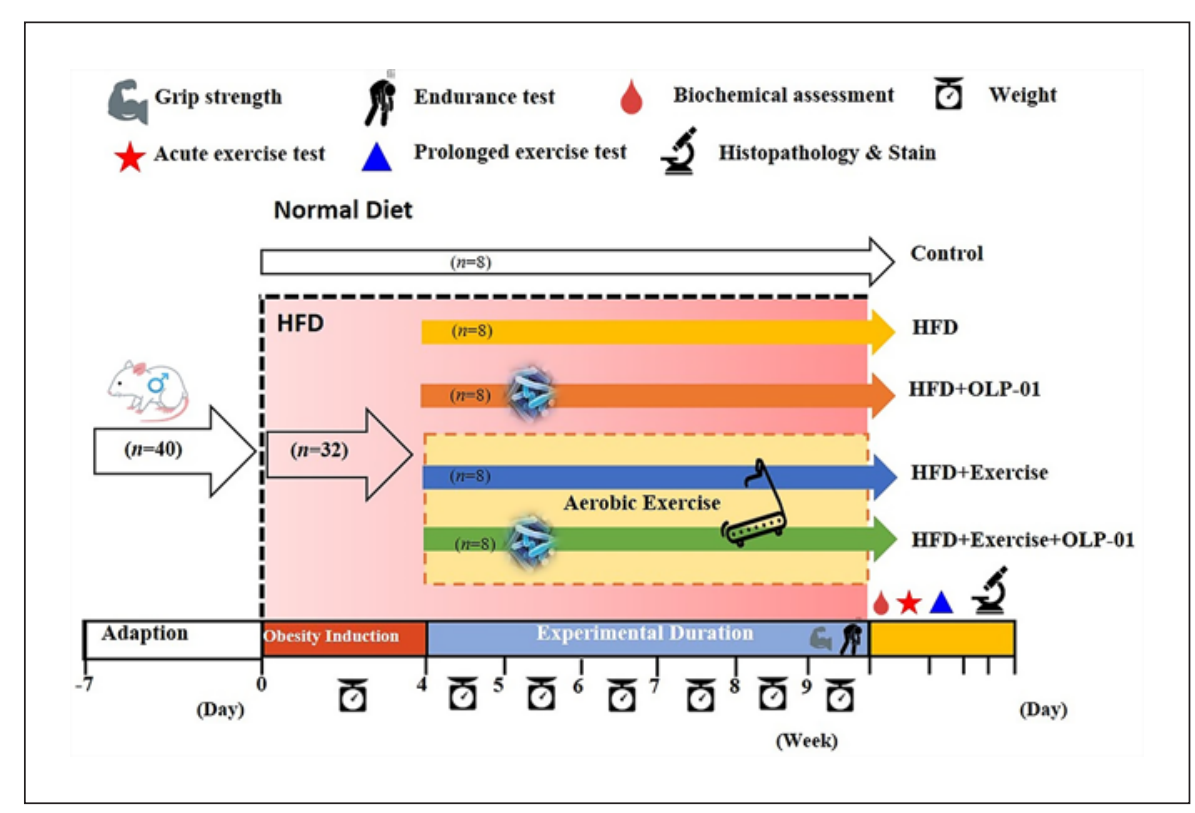

A previous study reported that probiotics can be also considered as ergogenic aids to improve exercise performance, oxidative stress, and inflammation in athletes [19]. Nutritional strategies and physical activities can be used as effective interventions for obesity management. However, limited studies have focused on the effects of a combination of probiotics and aerobic exercise on obesity. The effects of $B$. longum subsp. longum OLP-01 (OLP-01) isolated from an elite Olympic-level athlete on performance, oxidative stress, inflammation, and energy balance were previously reported [20]. Compared with nutritional strategies or exercise alone, a combination of nutritional strategies and aerobic exercise considerably improved body weight and adiposity parameters in a study [21]. Probiotics can be considered a nutritional supplement with multiple functional activities and systematic effects. Considering these findings, we investigated the combined use of OLP-01 probiotics and programmed aerobic treadmill exercise with respect to (1) its effects on obesity-related indexes and (2) its physiological effects in a murine model with high-fat diet (HFD)-induced obesity.

\section{Materials and Methods}

\section{Experimental Design}

Specific pathogen-free ICR (Institute of Cancer Research)strain mice ( 5 weeks old) purchased from BioLASCO (Yilan, Taiwan) were used. The mice were kept in a room with a 12-h light/ dark cycle at a temperature of $23 \pm 2{ }^{\circ} \mathrm{C}$ and $50-60 \%$ humidity. The health status and behavior of the animals were monitored by a veterinarian. Throughout the experimental duration, the mice in the different treatment groups were provided a standard chow diet (No. 5001; PMI Nutrition International, Brentwood, MO, USA) as well as HFD and sterilized water ad libitum, and their dietary and body weights were regularly recorded to evaluate their energy intake and growth. The chow diet was composed of $3.35 \mathrm{kcal} / \mathrm{g}$, with $28.5 \%$ protein, $13.4 \%$ fat, and $58.1 \%$ carbohydrates. The HFD was based on the chow diet, except that it was supplemented with $0.2 \%$ (wt/wt) cholesterol and 10\% (wt/wt) lard (both Sigma-Aldrich, St. Louis, MO, USA); it was composed of $3.92 \mathrm{kcal} / \mathrm{g}$, with $21.96 \%$ protein, $33.37 \%$ fat, and $44.67 \%$ carbohydrates.

After acclimation for 7 days, the animals were randomly assigned to the control (standard chow diet without exercise and probiotics), HFD, HFD with exercise (HFD + Ex), HFD with probiotics (HFD + OLP), and HFD with exercise and probiotics (HFD + Ex + OLP) groups. No significant difference was observed between the body weights of the mice in the different groups at the beginning and end of the obesity induction phase of 4 weeks. After induction, the mice in the different groups received the treadmill exercise protocol and/or probiotic supplementation for 5 weeks (Fig. 1). After the intervention, the physical fitness of the mice was evaluated by assessing their exhaustive swimming ability and forelimb grip strength. The glucose tolerance and biochemical indexes of mice undergoing acute and prolonged exercise challenges were also evaluated. All animal experimental procedures were reviewed by the Institutional Animal Care and Use Committee (IACUC) of National Taiwan Sport University, and the guidelines of protocol IACUC-10801 were approved by the Ethics Committee of IACUC.

\section{Probiotic Isolation and Identification}

OLP-1 was isolated from the feces of an elite weightlifting athlete; the feces were collected during the period of optimized training periodization when the athlete had their highest physical fit- 
ness capacities and the strength for Olympic competition. The optimal conditions for the feces collection (during the special preparatory phase in training periodization) are high intensity but relatively low training volume to ensure physiological adaptations and competitive capacities. The athlete's feces were series-diluted with phosphate-buffered saline and spread on selective BIM-25 medium with an anaerobic culture at $37^{\circ} \mathrm{C}$. After several selections and purification, a single colony was obtained, and its 16sRNA were amplified using the g-Bifid-F (5'-CTCCTGGAAACGGGTGG-3') and g-Bifid-R (5'-GGTGTTCTTCCCGATATCTACA- $3^{\prime}$ ) pair primer. The amplicons were further sequenced for microbial species identification. OLP-01 was cultivated, quantified, and maintained by Glac Biotech Co., Ltd. (Tainan, Taiwan). The Food Industry Research and Development Institute (Hsinchu, Taiwan) further identified and confirmed the isolated strain. The lyophilized powder was adjusted at a concentration of $1.07 \times 10^{11}$ $\mathrm{CFU} / \mathrm{g}$, and the aliquots of lyophilized powder were refrigerated at $-20^{\circ} \mathrm{C}$ until use. The dose of OLP-01 used in the current study $\left(1.03 \times 10^{10} \mathrm{CFU} / \mathrm{kg}\right)$ was based on the dose used in our previous study [20], and it was administered for 5 weeks through oral gavage. Mice in the other groups, namely the control, HFD, and exercise treatment groups, also received the same volume of saline according to their body weight $(10 \mathrm{~mL} / \mathrm{kg})$.

\section{Aerobic Exercise Training}

A motor-driven treadmill (Model MK-680; Muromachi Kikai, Tokyo, Japan) was used for the 5-week aerobic exercise training, and animal motivation was induced by an electric shock grid under veterinarian surveillance. The mice were initially acclimated to running at a speed of $10 \mathrm{~m} / \mathrm{min}$ for 2 days prior to the training protocol. The speed was increased to $12 \mathrm{~m} / \mathrm{min}$, and the training was conducted for $35 \mathrm{~min} /$ day in the first week, after which the speed was increased by $2 \mathrm{~m} / \mathrm{min}$ every week until the fifth week, when the mice were required to run at a speed of $20 \mathrm{~m} / \mathrm{min}$. The slope of the treadmill was also elevated from 0 to $5 \%$ in the third week and maintained at $10 \%$ for the last 2 weeks of the experiment.

\section{Physical Activities}

The forelimb grip strength and exhaustive swimming ability of the mice were evaluated to assess their anaerobic and aerobic capacities. Grip strength was assessed using a low-force testing system (Model-RX-5; Aikoh Engineering, Nagoya, Japan). The procedures and definitions of the physical activities were detailed in our previous study [20].

\section{Peripheral Fatigue-Associated Biochemical Variables}

Biochemical indexes can be used to indicate exercise-induced peripheral fatigue to, in turn, indicate the mice's physiological adaptations. The present protocol of acute and prolonged exercise challenge was a slight modification of the protocol employed in our previous study [20]. In the acute exercise challenge, mice blood was sampled at 3 time points: before swimming, immediately after swimming for $10 \mathrm{~min}$, and after the subsequent 20 -min rest inter$\mathrm{val}$, for analysis of their lactate profile. The prolonged exercise protocol included swimming for $90 \mathrm{~min}$, and blood was collected immediately after $60 \mathrm{~min}$ of rest and analyzed for creatine kinase (CK), lactate dehydrogenase (LDH), aspartate aminotransferase (AST), and alanine aminotransferase (ALT) levels. The blood samples were assessed using an autoanalyzer (Hitachi 7060; Hitachi, Tokyo, Japan).

Exercise and Probiotics for Diet-Induced Obesity

\section{Oral Glucose Tolerance Test}

This assay was performed at the end of the 5-week HFD/exercise/probiotics intervention. Mice were fasted for $12 \mathrm{~h}$ and then orally administered $1.0 \mathrm{~g}$ of glucose per $\mathrm{kg}$ of body weight. The blood glucose from the blood samples $(0.6 \mu \mathrm{L})$ collected at the indicated time points was measured using a glucometer (Accu$\mathrm{Chek}^{\circledR}$; Roche, Taipei, Taiwan) to determine plasma glucose concentration [22].

\section{Clinical Biochemical Parameters}

The mice were euthanized by asphyxiation with $95 \% \mathrm{CO}_{2}$, and their blood samples were immediately collected through cardiac puncture. The serum was separated through centrifugation at $1,000 \mathrm{~g}$ for $15 \mathrm{~min}$ at $4{ }^{\circ} \mathrm{C}$ after ensuring complete clotting. The separated serum was used to analyze the clinical biochemical parameters of AST, ALT, CK, glucose, blood urea nitrogen (BUN), creatinine (CREA), uric acid (UA), albumin, triglyceride, total cholesterol, high-density lipoprotein, and low-density lipoprotein using an autoanalyzer (Hitachi 7060; Hitachi, Tokyo, Japan).

\section{Body Composition and Histology}

After the mice were sacrificed, their visceral organs of interest - namely the liver, kidneys, skeletal muscle (gastrocnemius and soleus), and white adipocyte tissue (epididymal fat pad and perirenal fat) - were precisely excised and weighed to determine the body composition of the mice. The organs were preserved in $10 \%$ formalin for histopathological analysis with hematoxylin and eosin staining.

\section{Statistical Analysis}

Data were represented in terms of the mean $\pm S D$, and the final numbers were rounded to the appropriate numerical presentation. All variables were qualified using the Kolmogorov-Smirnov test before the parametric test, and the Kruskal-Wallis test was applied to variables without normal distributions. Statistically significant differences in physical activity, biochemistry, body weight, and body composition between the groups were analyzed using oneway ANOVA, and mixed two-way ANOVA was applied to evaluate the effects on lactate profiles, growth curve, and glucose tolerance to verify the main and interaction effects on these factors. Significant differences were further validated using a post hoc Duncan test, and the data were considered statistically significant when the probability of a type I error was $<0.05$. Significant differences between treatments are represented by various subscript Roman letters ( $a, b, c, d)$ with the compact letter display method in figures and tables.

\section{Results}

\section{Effects of Exercise and Probiotics on Growth Curve in HFD-Induced Obesity}

The mice in the 5 groups weighed approximately $31 \mathrm{~g}$, and no significant difference was found between the weights of the mice in the different groups $(F[4.35]=$ $0.275, p=0.892$ ) at the beginning of the experiment. An HFD was administered to mice in the HFD groups (HFD, 
Fig. 2. Effects of the aerobic exercise and probiotic interventions on growth. Obesity was induced by an HFD for 4 weeks, and this was followed by the indicated treatments over 5 weeks. Data are the mean \pm SD per group. Treatments with different letters $(\mathrm{a}-\mathrm{c})$ are significantly different at $p<0.05$.

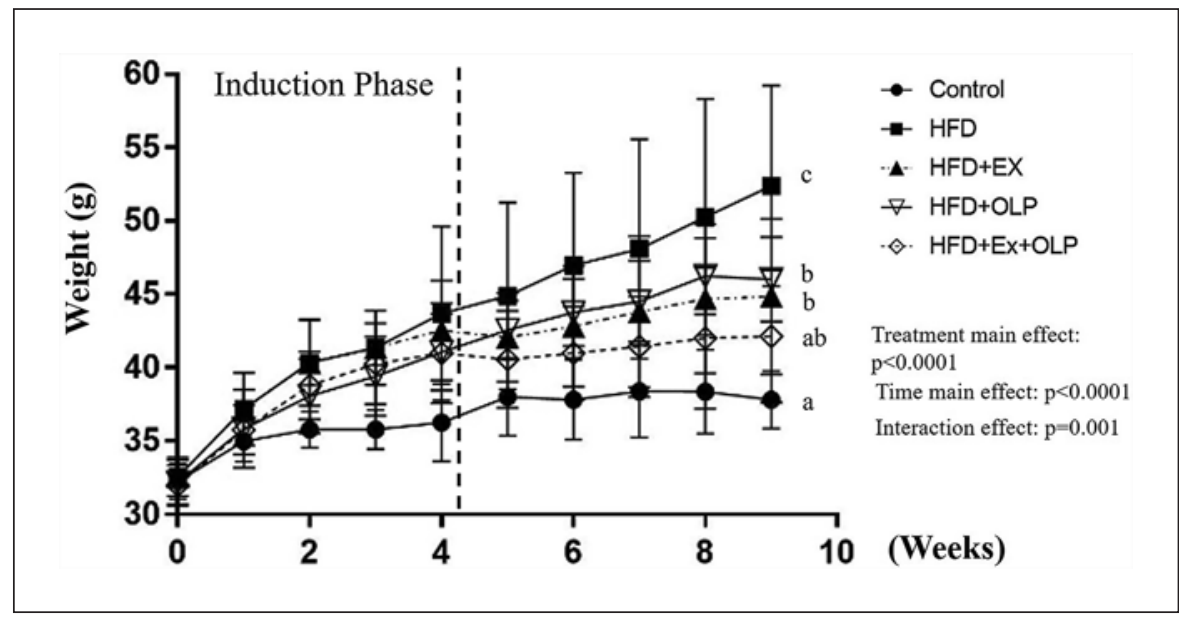

Table 1. Effects of exercise and probiotics on body composition and diet intake

\begin{tabular}{|c|c|c|c|c|c|}
\hline Characteristic & Control & HFD & $\mathrm{HFD}+\mathrm{Ex}$ & HFD + OLP & $\mathrm{HFD}+\mathrm{Ex}+\mathrm{OLP}$ \\
\hline Liver, g & $2.14 \pm 0.26$ & $2.14 \pm 0.28$ & $2.15 \pm 0.38$ & $2.06 \pm 0.16$ & $2.14 \pm 0.29$ \\
\hline Muscle, $g$ & $0.32 \pm 0.04$ & $0.31 \pm 0.05$ & $0.32 \pm 0.04$ & $0.29 \pm 0.03$ & $0.33 \pm 0.02$ \\
\hline Kidney, g & $0.71 \pm 0.04$ & $0.66 \pm 0.07$ & $0.66 \pm 0.10$ & $0.71 \pm 0.06$ & $0.69 \pm 0.08$ \\
\hline Heart, g & $0.24 \pm 0.17$ & $0.21 \pm 0.03$ & $0.22 \pm 0.03$ & $0.22 \pm 0.02$ & $0.22 \pm 0.03$ \\
\hline $\mathrm{EPF}, \mathrm{g}$ & $0.50 \pm 0.36^{\mathrm{a}}$ & $2.65 \pm 0.81^{\mathrm{c}}$ & $1.97 \pm 0.67^{\mathrm{b}}$ & $1.90 \pm 0.46^{\mathrm{b}}$ & $1.06 \pm 0.36^{\mathrm{a}}$ \\
\hline Perirenal fat, $g$ & $0.18 \pm 0.08^{\mathrm{a}}$ & $1.18 \pm 0.34^{\mathrm{d}}$ & $0.88 \pm 0.40^{\mathrm{c}}$ & $0.78 \pm 0.13^{\mathrm{b}, \mathrm{c}}$ & $0.51 \pm 0.23^{b}$ \\
\hline Energy intake, calorie/mouse/day & $13.4 \pm 0.64^{\mathrm{a}}$ & $15.6 \pm 0.65^{\mathrm{b}}$ & $15.5 \pm 0.91^{\mathrm{b}}$ & $15.8 \pm 1.04^{\mathrm{b}}$ & $15.7 \pm 0.83^{\mathrm{b}}$ \\
\hline
\end{tabular}

Data are expressed as the mean \pm SD in each group. Values in the same row with different superscript letters (a, b, c) differ significantly, $p<0.05$, by one-way analysis of variance (ANOVA) and Kruskal-Wallis test. Muscle, gastrocnemius and soleus; EFP, epididymal fat pad. Control and HFD mean the normal chow diet and high fat diet administration, respectively.

HFD + Ex, HFD + OLP, and HFD + Ex + OLP groups) for 4 weeks to induce obesity, whereas mice in the control group were fed the normal diet (control). After the induction of obesity, a significant difference was observed in the weights of the mice between the different groups $(F[4.35]=4.43, p=0.005)$. Mice in the HFD groups had $10 \%$ more body weight than mice in the control group; no significant difference was observed between the weights of the mice in the different HFD groups. The mice were then subjected to exercise and/or administered probiotics for 5 weeks; significant differences were observed in the main and interaction effects of the type and duration of treatments administered to the mice $(F[4.140]=38.55, p$ $<0.0001 ; F[4.35]=40.1, p<0.0001 ; F[16.140]=5.95, p<$ 0.0001 , respectively). Significant differences were observed in the simple main effects between the groups in the ninth week $(F[4.35]=10.69, p<0.0001)$. The body weights of mice in the control, HFD + Ex, HFD + OLP, and HFD + Ex + OLP groups were significantly lower than those of mice in the HFD groups. The body weights of mice in the HFD + EX, and HFD + OLP groups were significantly higher than those of mice in the control group, and the body weights of mice in the control and HFD + Ex + OLP groups did not significantly differ (Fig. 2). The body weight of the HFD group was significantly higher than those of the HFD + Ex and HFD + EX + OLP groups ( $p=0.003$ and $p<0.0001$, respectively), but no significant difference was found between the HFD + Ex and HFD + EX + OLP groups. The daily energy intake of mice in the HFD groups was significantly higher than that of mice in the control group (Table 1). Therefore, the effects of exercise and/or OLP-01 probiotics can modulate HFD-induced weight gain, and the physiological effects on functional activities can be further identified as detailed below. 
Fig. 3. Effect of a 5-week exercise and probiotic intervention on exhaustive swimming time. Columns with different letters (a-d) are significantly different at $p<0.05$.

Fig. 4. Effect of a 5-week exercise and probiotic intervention on grip strength $(\mathbf{A})$ and relative strength $(\mathbf{B})$. Data are the mean \pm $\mathrm{SD}$, and columns with different letters (ac) are significantly different at $p<0.05$.

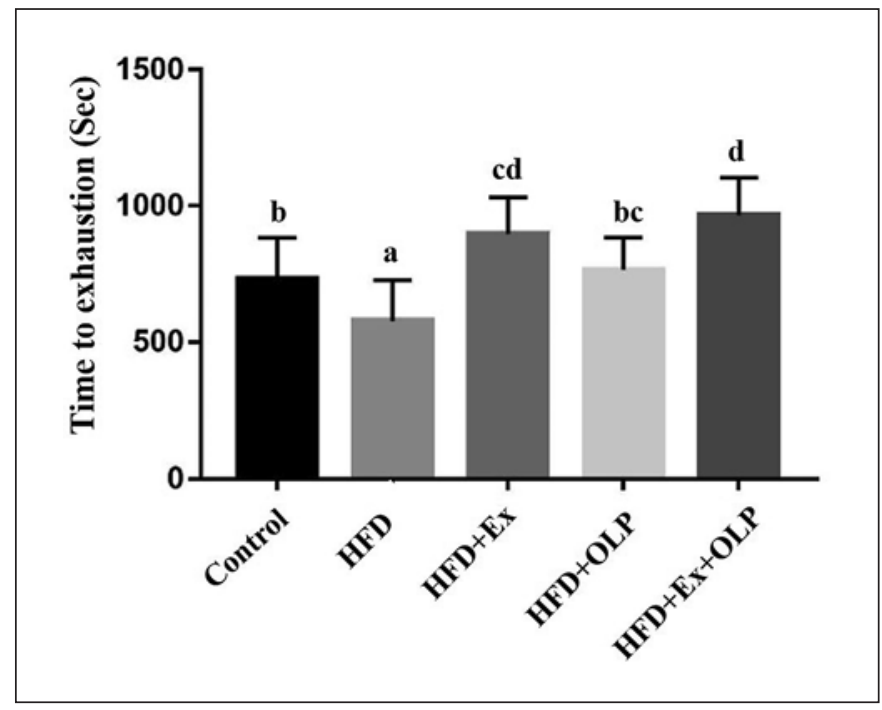

(A)

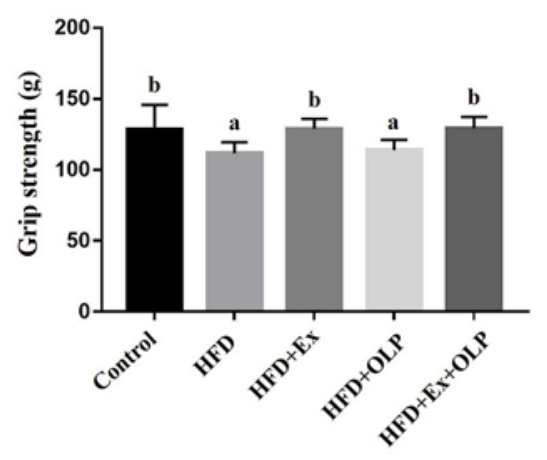

(B)

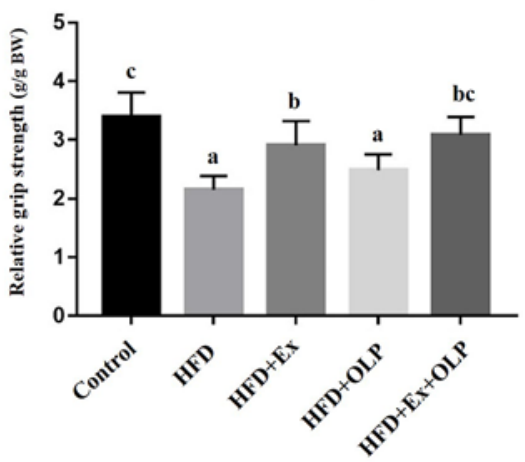

Effects of Exercise and Probiotics on Physical Activities of Mice with HFD-Induced Obesity

We evaluated the physical activity of the mice based on exhaustive swimming and grip strength tests, doing so to evaluate the effects of exercise and/or probiotics on HFDinduced obesity. Significant differences were observed between mice in the different groups with respect to endurance in the exhaustive swimming test $(F[4.35]=9.5$, $p<0.0001)$. The endurance of mice in the HFD group was significantly lower than that of mice in the other groups; in addition, mice in the HFD + Ex and HFD + Ex + OLP groups exhibited considerably higher endurance levels than mice in the control group (Fig. 3).

Grip strength has been reported to be significantly and positively correlated with anthropometric factors - such as forearm circumference, hand length, and weight [23]. The absolute and relative grip strengths, calibrated by individual weights, of mice in the different treatment groups are presented in Figure 4. Significant differences were observed between the different groups with respect to grip strength $(F[4.35]=7.7, p<0.0001)$. Compared with mice in the control, HFD + Ex, and HFD + Ex + OLP groups, those in the HFD and HFD + OLP groups exhibited significantly decreased grip strength. After calibration with reference to the body weight of the mice, significant differences were observed between mice in the different groups with respect to relative grip strength $(F[4.35]=$ $17.3, p<0.0001)$. The relative grip strength of the mice in the control group was significantly greater than that of mice in the HFD, HFD + Ex, and HFD + OLP groups. Compared with mice in the HFD and HFD + OLP groups, those in the HFD + Ex group exhibited greater grip strength. No significant difference was observed in grip strength between the mice in the HFD + Ex + OLP group and the mice in the control and HFD + Ex groups. 
Table 2. The effects of exercise and probiotics on lactate profiles during acute exercise challenge

\begin{tabular}{|c|c|c|c|c|c|}
\hline Time point & Control & HFD & $\mathrm{HFD}+\mathrm{Ex}$ & HFD + OLP-01 & $\mathrm{HFD}+\mathrm{Ex}+\mathrm{OLP}-01$ \\
\hline \multicolumn{6}{|l|}{ Lactate, $\mathrm{mmol} / \mathrm{L}$} \\
\hline Before swimming (A) & $3.3 \pm 0.3^{\mathrm{a}}$ & $3.5 \pm 0.3^{\mathrm{a}}$ & $3.1 \pm 0.4^{\mathrm{a}}$ & $3.5 \pm 0.5^{\mathrm{a}}$ & $3.4 \pm 0.3^{\mathrm{a}}$ \\
\hline After swimming (B) & $4.4 \pm 0.3^{\mathrm{a}}$ & $5.6 \pm 0.7^{c}$ & $4.0 \pm 0.4^{\mathrm{a}}$ & $5.1 \pm 0.7^{b}$ & $4.4 \pm 0.4^{\mathrm{a}}$ \\
\hline After a 20 min rest $(C)$ & $3.4 \pm 0.4^{\mathrm{a}}$ & $4.3 \pm 0.8^{\mathrm{b}}$ & $3.1 \pm 0.4^{\mathrm{a}}$ & $3.4 \pm 0.4^{\mathrm{a}}$ & $3.3 \pm 0.4^{\mathrm{a}}$ \\
\hline \multicolumn{6}{|c|}{ Rate of lactate production and clearance } \\
\hline Production rate $=\mathrm{B} / \mathrm{A}$ & $1.32 \pm 0.1^{\mathrm{a}}$ & $1.61 \pm 0.2^{\mathrm{b}}$ & $1.28 \pm 0.1^{\mathrm{a}}$ & $1.47 \pm 0.1^{\mathrm{b}}$ & $1.31 \pm 0.1^{\mathrm{a}}$ \\
\hline Clearance rate $=(\mathrm{B}-\mathrm{C}) / \mathrm{B}$ & $0.22 \pm 0.11$ & $0.24 \pm 0.06$ & $0.22 \pm 0.16$ & $0.33 \pm 0.07$ & $0.24 \pm 0.08$ \\
\hline
\end{tabular}

The control, HFD, HFD + Ex, HFD + OLP-01, and HFD + Ex + OLP-01 groups were repeatedly sampled at indicated 3 time points for lactate metabolites assessment. The lactate production rate was calculated as lactate production after exercise divided by lactate production before exercise $(\mathrm{B} / \mathrm{A})$, and the clearance rate $([\mathrm{B}-\mathrm{C}] / \mathrm{B})$ was defined as lactate production difference between after exercise and after rest, divided by lactate production after exercise. Values in the same row with different superscript letters (a, b, $c)$ differ significantly, $p<0.05$, by one-way analysis of variance (ANOVA) and Kruskal-Wallis test. Control and HFD mean the normal chow diet and high fat diet administration, respectively.

Fig. 5. Effect of exercise and probiotic intervention on CK $(\mathbf{A}), \operatorname{LDH}(\mathbf{B}), \operatorname{AST}(\mathbf{C})$, and ALT (D) levels after an extended exercise challenge. The indicated 4 groups underwent 90-min swimming, and blood was sampled after 60 min of rest. Data are the mean $\pm \mathrm{SD}$, and columns with different letters $(\mathrm{a}-\mathrm{d})$ are significantly different at $p<$ 0.05 .
(A)

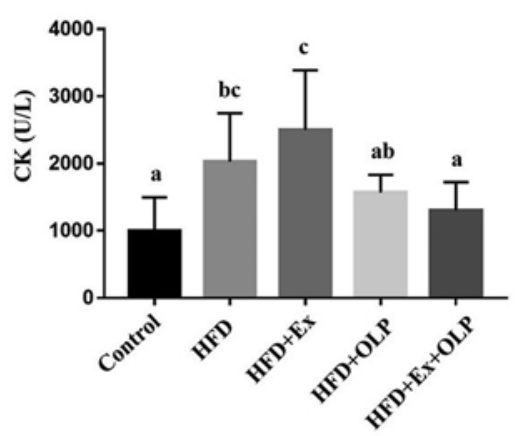

(C)

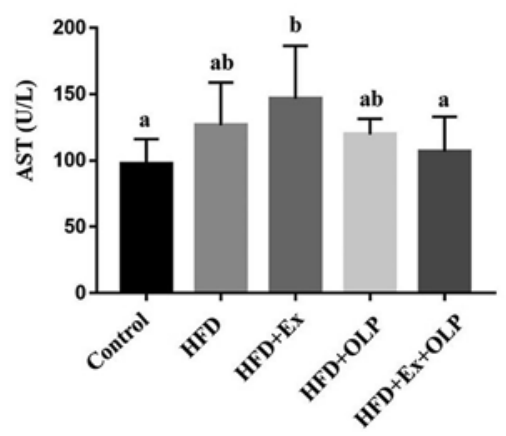

(B)

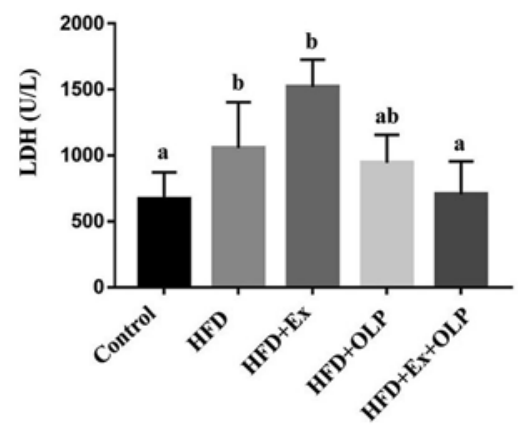

(D)

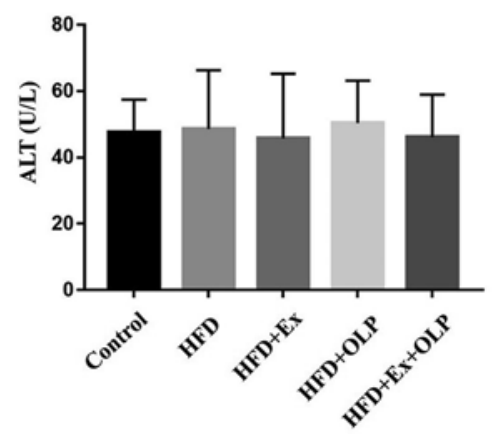

\section{Effects of Exercise and Probiotic Intervention on}

Fatigue-Associated Biochemistry

The extent of central and peripheral fatigue experienced after exercise varies according to the intensity, frequency, and duration of exercise. However, several biochemical parameters, such as lactate, CK, LDH, AST, and
ALT, can directly indicate the physiological responses after physical fatigue due to the altered energy metabolism and oxidative stress during exercise [24].

The lactate profile of the animals was examined during the acute exercise challenge, as shown in Table 2. A significant difference was observed not only in the main ef- 


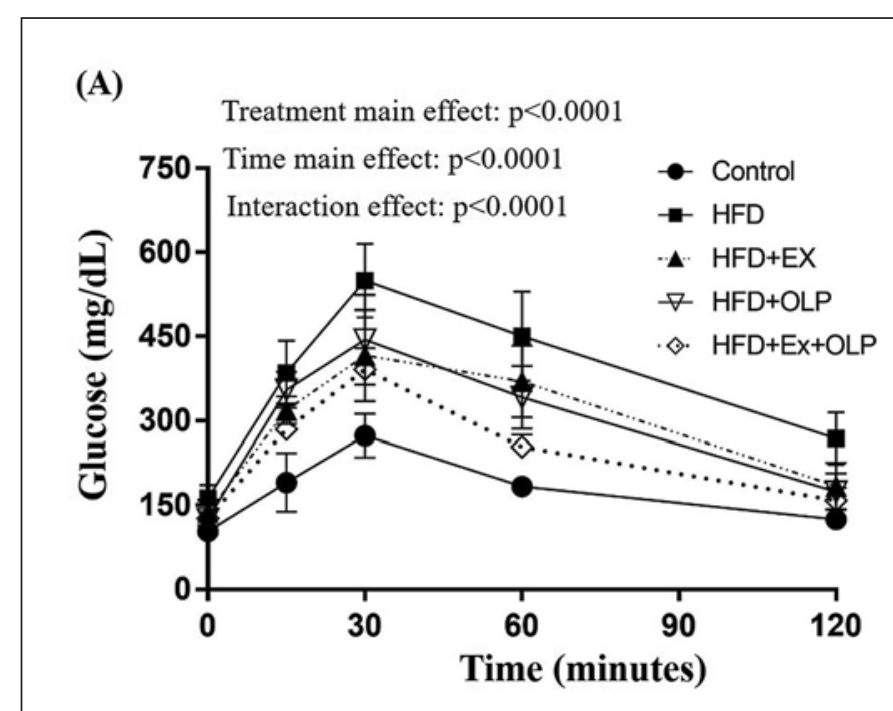

(B)

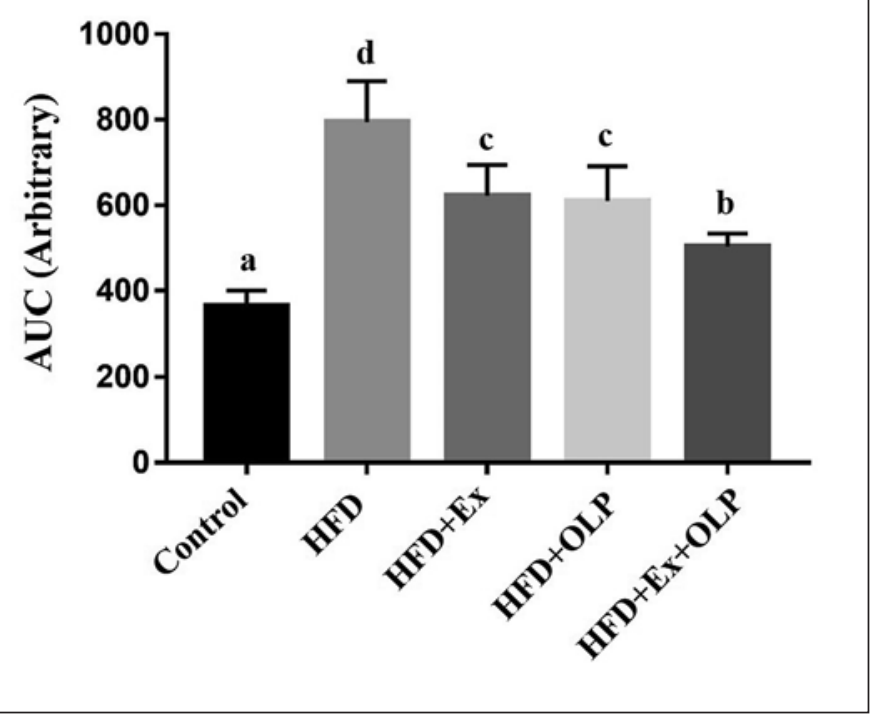

Fig. 6. Effect of exercise and probiotic intervention on glucose tolerance $(\mathbf{A})$, and area under curve (B) levels after extended exercise challenge. The oral glucose tolerance test was performed at the end of the study and at the indicated time points $(0,15,60,90$, and 120

min) immediately after $1 \mathrm{~g} / \mathrm{kg}$ (body weight) oral glucose supplementation for glucose analysis. Data are the mean \pm SD, and columns with different letters $(\mathrm{a}-\mathrm{d})$ are significantly different at $p<$ 0.05 .

fects $(F[4.35]=9.4, p<0.0001 ; F[2.70]=183, p<0.0001)$ but also in the interaction effects $(F[8.70]=5.90, p<$ $0.0001)$ of the type and duration of treatment. Significant differences were observed between the simple main effects observed immediately after exercise and after a 20min rest period (2 time points after exercise; $F[4.35]=$ $13.2, p<0.0001$ and $F[4.35]=1.59, p=0.001$, respectively). The lactate levels of mice in the HFD group were significantly higher than those of mice in the control group. Mice in the exercise and/or OLP-01 supplementation groups (HFD + Ex, HFD + OLP, and HFD + Ex + OLP) exhibited significantly lower lactate levels than mice in the HFD group. After exercise, mice in the HFD group still exhibited significantly higher lactate levels than mice in the other groups. Mice in the HFD + Ex and HFD + Ex + OLP groups had significantly lower lactate production rates than the mice in the HFD and HFD + OLP groups $(F[4.35]=7.42, p<0.0001)$; however, the groups did not significantly differ with respect to lactate clearance $(F[4.35]=1.74, p=0.162)$.

$\mathrm{CK}, \mathrm{LDH}, \mathrm{AST}$, and ALT levels were assessed for injuryassociated markers after prolonged exercise (Fig. 5). Significant differences were observed between mice in the different groups with respect to the levels of CK $(F[4.35]=$ $7.89, p<0.0001), \operatorname{LDH}(F[4.35]=5.11, p=0.002)$, and AST

$(F[4.35]=3.76, p=0.012)$ but not $\operatorname{ALT}(F[4.35]=0.13, p=$ 0.971). Compared with the control group, mice in the HFD group exhibited significantly elevated CK levels after prolonged exercise. Similarly, significantly higher CK levels were observed in mice in the HFD + Ex group - compared with those of mice in the control, HFD + OLP, and HFD + Ex + OLP groups. OLP-01 supplementation (HFD + Ex + OLP) significantly reduced CK levels, compared with those of mice in the HFD + Ex group $(p<0.0001)$. LDH levels did not significantly differ between mice in the HFD and HFD + Ex groups, and OLP-01 supplementation (HFD + Ex + OLP) significantly reduced LDH levels that were increased due to prolonged exercise - compared with those of mice in the HFD + Ex group $(p=0.001)$. Similar results were observed in the AST index. Compared with mice in the control and HFD + Ex + OLP groups, those in the HFD + Ex group exhibited significantly increased AST levels after prolonged exercise, and the HFD + Ex + OLP group exhibited significantly reduced AST levels - compared with those of mice in the HFD + Ex group $(p=0.007)$.

\section{Effect of Exercise and Probiotics on Glucose Tolerance} Profiles

Mice were subjected to exercise and/or OLP-01 administration for 5 weeks and were fasted for $12 \mathrm{~h}$ prior to 
Table 3. Effects of exercise and probiotic intervention on clinical biochemical analysis

\begin{tabular}{|c|c|c|c|c|c|}
\hline Parameter & Control & HFD & $\mathrm{HFD}+\mathrm{Ex}$ & HFD + OLP & $\mathrm{HFD}+\mathrm{Ex}+\mathrm{OLP}$ \\
\hline GLU, mg/dL & $170 \pm 15^{\mathrm{a}}$ & $233 \pm 22^{d}$ & $203 \pm 22^{b c}$ & $218 \pm 23^{\mathrm{cd}}$ & $191 \pm 21^{b}$ \\
\hline BUN, mg/dL & $21.1 \pm 1.9^{\mathrm{a}}$ & $35.9 \pm 2.1^{\mathrm{c}}$ & $31.1 \pm 1.6^{\mathrm{b}}$ & $33.6 \pm 5.2^{\mathrm{bc}}$ & $32.4 \pm 2.6^{\mathrm{b}}$ \\
\hline CREA, mg/dL & $0.41 \pm 0.03^{\mathrm{a}}$ & $0.50 \pm 0.04^{\mathrm{c}}$ & $0.45 \pm 0.03^{\mathrm{b}}$ & $0.44 \pm 0.03^{\mathrm{b}}$ & $0.41 \pm 0.04^{\mathrm{a}}$ \\
\hline $\mathrm{CK}, \mathrm{U} / \mathrm{L}$ & $170 \pm 33^{\mathrm{a}}$ & $187 \pm 28^{\mathrm{a}}$ & $271 \pm 66^{\mathrm{b}}$ & $191 \pm 48^{\mathrm{a}}$ & $217 \pm 44^{\mathrm{a}}$ \\
\hline $\mathrm{UA}, \mathrm{mg} / \mathrm{dL}$ & $1.60 \pm 0.2^{\mathrm{a}}$ & $2.51 \pm 0.6^{c}$ & $2.31 \pm 0.8^{\mathrm{bc}}$ & $2.02 \pm 0.3^{\mathrm{abc}}$ & $1.89 \pm 0.2^{\mathrm{ab}}$ \\
\hline AST, U/L & $104 \pm 24$ & $106 \pm 26$ & $105 \pm 25$ & $111 \pm 28$ & $108 \pm 33$ \\
\hline ALT, U/L & $49 \pm 7$ & $46 \pm 13$ & $46 \pm 10$ & $47 \pm 12$ & $44 \pm 15$ \\
\hline $\mathrm{ALB}, \mathrm{g} / \mathrm{dL}$ & $2.6 \pm 0.4$ & $2.9 \pm 0.1$ & $2.9 \pm 0.2$ & $2.9 \pm 0.2$ & $2.9 \pm 0.3$ \\
\hline $\mathrm{TC}, \mathrm{mg} / \mathrm{dL}$ & $193 \pm 14^{\mathrm{a}}$ & $361 \pm 58^{c}$ & $260 \pm 55^{b}$ & $256 \pm 74^{b}$ & $237 \pm 23^{\mathrm{ab}}$ \\
\hline $\mathrm{TG}, \mathrm{mg} / \mathrm{dL}$ & $174 \pm 21^{\mathrm{a}}$ & $299 \pm 93^{b}$ & $221 \pm 84^{\mathrm{a}}$ & $231 \pm 65^{\mathrm{ab}}$ & $178 \pm 75^{\mathrm{a}}$ \\
\hline $\mathrm{HDL}, \mathrm{mg} / \mathrm{dL}$ & $109 \pm 19^{a}$ & $203 \pm 40^{c}$ & $153 \pm 24^{b}$ & $146 \pm 50^{\mathrm{ab}}$ & $136 \pm 35^{\mathrm{ab}}$ \\
\hline $\mathrm{LDL}, \mathrm{mg} / \mathrm{dL}$ & $34 \pm 6^{\mathrm{a}}$ & $77 \pm 18^{\mathrm{c}}$ & $56 \pm 12^{\mathrm{b}}$ & $51 \pm 10^{\mathrm{b}}$ & $46 \pm 13^{\mathrm{ab}}$ \\
\hline
\end{tabular}

Data are expressed as the mean \pm SD in each group. Values in the same row with different superscript letters (a, b) differ significantly, $p<0.05$, by one-way ANOVA and Kruskal-Wallis test; AST, aspartate aminotransferase; ALT, alanine transaminase; NH3, ammonia; CK, creatine kinase; GLU, glucose; CREA, creatinine; BUN, blood urea nitrogen; UA, uric acid; TC, total cholesterol; TG, triacylglycerol; ALB, albumin; HDL, high-density lipoprotein; LDL, low-density lipoprotein. Control and HFD mean the normal chow diet and high fat diet administration, respectively.

the oral glucose tolerance test. Blood was collected from the tail vein of the mice at indicated time points, and the status of glucose tolerance was evaluated. Mixed two-way ANOVA revealed significant main $(F[4.140]=30.8, p<$ $0.0001 ; F[4.35]=40.8, p<0.0001)$ and interaction effects of the type and duration of treatment on the glucose tolerance of mice $(F[4.140]=7.01, p<0.0001$; Fig. 6). The results indicated that the main treatment effect on mice in the HFD + Ex + OLP group was significantly lower than that on mice in the HFD + Ex, HFD + OLP, and HFD groups but higher than the effects on mice in the control group. The areas under the curve of the indicated treatments were calculated from individual glucose profiles, and a significant difference was observed between the mice in the different groups $(F[4.35]=43.1, p<0.0001)$. The glucose intolerance of mice in the HFD group was significantly higher than that of mice in other groups, and mice in the HFD + Ex + OLP group exhibited improved glucose tolerance, compared with mice in the HFD + Ex and HFD + OLP groups.

\section{Effect of Exercise and Probiotics on Biochemical \\ Parameters}

After the mice were sacrificed at the end of the experiment, blood was collected through cardiac puncture and further analyzed for various biochemical parameters. As indicated in Table 3, the glucose and BUN indexes sig- nificantly differed between the groups $(F[4.35]=29.8$, $p<0.0001$ and $F[4.35]=11.2, p<0.0001$, respectively). The glucose and BUN levels were significantly higher in mice in the HFD group than in those in the control group and were significantly lower in mice in the HFD + Ex and HFD + Ex + OLP groups than in mice in the HFD group. The CK and UA levels also differed significantly between the groups $(F[4.35]=5.96, p=0.001$ and $F[4.35]=3.987$, $p=0.009$, respectively). The HFD + Ex + OLP group exhibited significantly decreased levels of $\mathrm{CK}$ and UA, which were elevated by the HFD and/or exercise intervention (HFD and HFD + Ex). Mice in the HFD group exhibited increased levels of TC, TG, HDL, and LDL, and mice in the HFD + Ex + OLP group exhibited significantly reduced levels for these indexes after 5 weeks. The other indexes, AST, ALT, ALB, and CREA, did not significantly differ between the groups.

\section{Effects of Exercise and Probiotics on Body Composition and Histology}

The body compositions of the mice are presented in Table 1. No significant differences were observed in the liver, muscle, kidney, and heart tissues between mice in the different groups $(p>0.05)$. The amount of white adipocyte tissue (epididymal fat and perirenal fat) differed significantly among the groups $(F[4.35]=17.7, p<0.0001$ and $F[4.35]=16.3, p<0.0001$, respectively). The HFD 


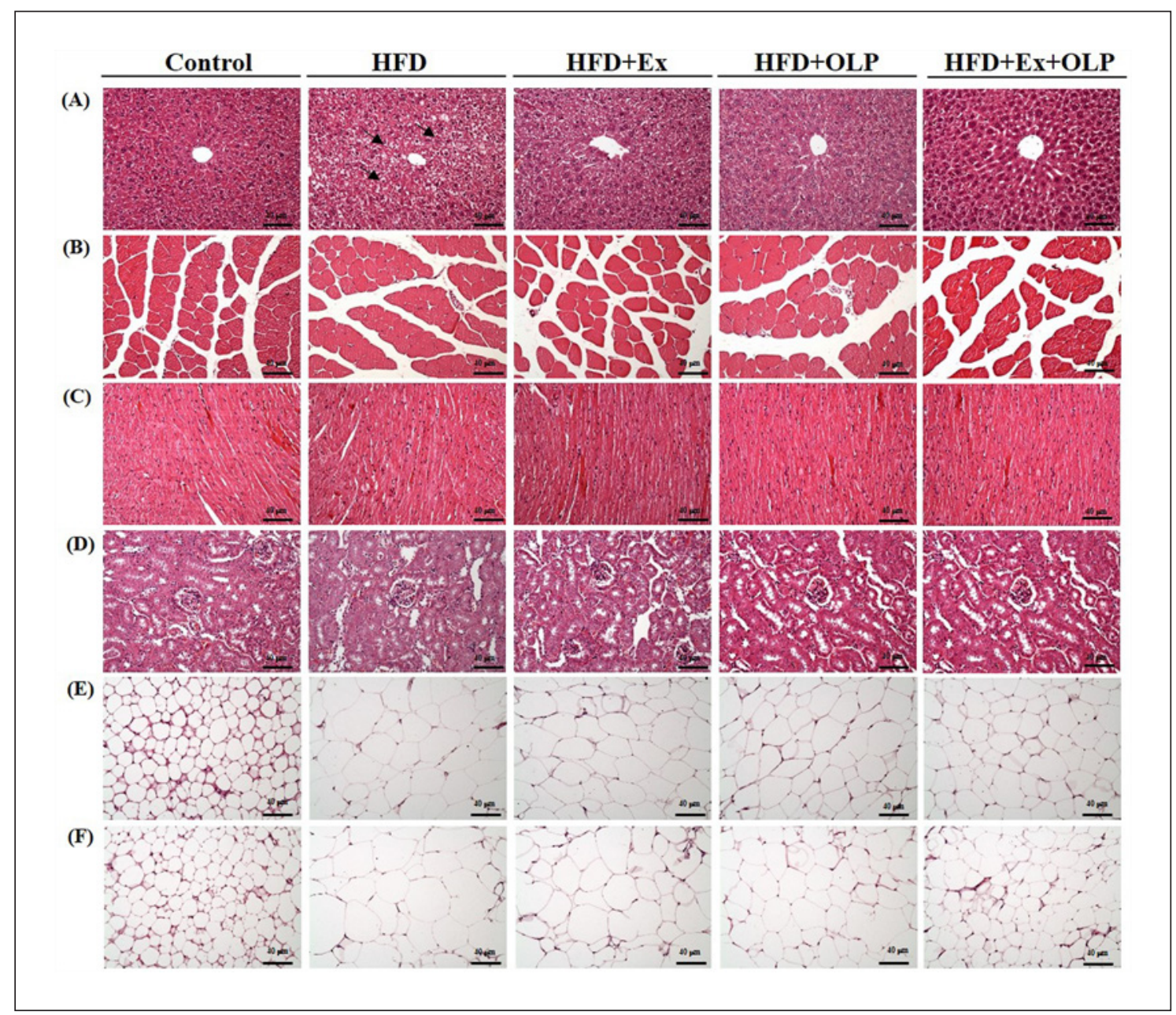

Fig. 7. Effect of exercise and probiotic intervention on histopathology. At the end of study, the indicated liver (A), muscle (B), heart (C), kidney (D), perirenal fat (E), and epididymal fat (F) tissues were stained with hematoxylin and eosin (H\&E), and the specimens were photographed under a light microscope (H\&E stain, magnification: $\times 200$; bar, $40 \mu \mathrm{m})$.

group had the most epididymal fat and perirenal fat tissues. Exercise or OLP supplementation also decreased the weight of fat tissues; however, significant benefits were observed in the different tissues of the HFD + Ex + OLP group compared with the HFD + Ex group.

The tissues (liver, muscle, heart, kidney, epididymal fat pads, and perirenal fat pads) of the mice were evaluated for potential histopathological changes due to exercise and probiotic supplementation (Fig. 7). The liver tissues of mice in the HFD group exhibited mild microvesicular steatosis along with glycogen (indicated by arrows in Fig. 7) surrounding the pericentral areas. Exercise training and/or probiotic intervention significantly reduced steatosis severity. No significant changes were ob- served in the liver structures, including the sinusoids and hepatic cords, between mice in the different groups after the indicated treatments. No histologic changes - such as Zenker's degeneration, hyperplasia, or inflammatory cell infiltration - were observed in the heart or skeletal muscle tissues of mice in any of the groups. The structure of renal tubules or glomeruli did not differ between mice in the control and HFD groups. Adipocytes containing large concentrations of lipids in the white adipose tissue were observed in the HFD group but not in the control group. Notably, a trend toward smaller adipocytes in white adipocyte tissues was discovered in the HFD + OLP1, HFD $+\mathrm{EX}$, and HFD + Ex + OLP groups. 


\section{Discussion}

The role of OLP-01 probiotics in the mitigation of oxidative stress and inflammation induced by exercise training has been reported in our previous study [20]. Obesity is likely associated with oxidative stress, inflammation, and dyslipidemia, and the impaired glucose tolerance observed in obesity can also, in turn, promote the progression of obesity. Probiotics and exercise have been reported to improve the physiological factors associated with obesity. In the current study, we demonstrated that compared with exercise or probiotics alone, the combination of OLP-01 probiotics and exercise had a stronger positive influence on physical endurance, weight loss, physiological adaptations, glucose tolerance, and histology in an HFD-induced murine obesity model; additionally, OLP01 mitigated exercise-induced oxidative stress under obesity conditions, enabling superior health maintenance.

In a previous study that involved the investigation of multiple probiotics, 5 different Bacillus species were applied to an HFD-induced obesity model; the isolates substantially reduced body weight and fat mass, improved glucose tolerance, and reduced hepatic steatosis through the regulation of gene expression related to lipid uptake and lipogenesis [25]. Our previous study also reported that the exercise training protocol used in the current study mitigated body weight increases and resulted in higher exercise performance [20]. Thus, the exercise protocol potentially exerts functional effects on physiological activities. In addition, in the present study, the exercise protocol attenuated weight gain in mice with HFD-induced obesity, and OLP-01 probiotics significantly reduced increases in weight in these mice. Moreover, OLP01 probiotics and exercise more greatly aided weight management when applied jointly than singly (Fig. 2).

A previous study investigating the relationship between obesity and physical activity revealed that a $16.3 \%$ weight reduction improved inspiratory capacity and physical function by 45.8 and $27 \%$, respectively [26]. Being overweight or obese is associated with higher risks of comorbidities, gait pattern modifications, and changes in the musculoskeletal structure and even absolute and relative energy expenditures [27]. We observed that the endurance capacity of mice in the HFD group was significantly lower than that of mice in the control group; mice in the HFD + Ex and HFD + Ex + OLP groups exhibited significantly higher endurance capacity than mice in the control and HFD groups (Fig. 3). A previous study also showed that earlier onset of obesity was associated with lower hand grip strength when covariates were controlled
[28]. Interventions such as vibration training can also significantly increase grip strength in people with obesity [29]. In the present study, the treadmill exercise involved a $5-10 \%$ slope, along with uphill resistance training. Mice in the HFD group had significantly lower grip strength than mice in the control group, and it also demonstrated that the uphill treadmill training significantly aided the recovery of grip strength with comparison of the HFD + EX and HFD groups. Thus, the exercise intervention helped improve the aerobic exercise capacity and grip strength of the mice with HFD-induced obesity.

The levels of lactate, CK, LDH, AST, and ALT represent the energy balance of the exercise metabolites and the injury caused by oxidative stress during exercise, and these levels depend on the intensity and duration of the exercise [30]. A previous study reported that (1) the level of monocarboxylate transporter 2 , which is responsible for lactate uptake, was significantly decreased in the liver of individuals with HFD-induced obesity and (2) blood lactate was quickly accumulated during incremental exercise followed by poorer exercise performance [31]. The treadmill exercise training mitigated the significant lactate accumulation and production rate in the HFD group, and OLP-01 probiotics can also ameliorate deteriorations to the lactate profile (Table 2), consistent with our previous study [20]. Moreover, obesity can significantly exacerbate the oxidative stress caused by an increased production of lipid hydroperoxides and malondialdehyde, observed immediately after resistance and aerobic exercises [32]. Therefore, the levels of enzymes relevant to oxidative stress, such as LDH, CK, and AST, were significantly elevated immediately after acute exercise, which can be significantly reduced using a combination of OLP-01 probiotics and exercise. The use of an assistive device can also mitigate the muscular damage caused by physical activities in individuals with obesity [33]. Thus, the present study reveals that the use of probiotics benefits physiological adaptation for people with obesity and when used in conjunction with exercise training.

Innate immunity in the course of obesity can be activated and mediated by metabolic signals, followed by the stimulation of downstream pathways - including I $\mathrm{KB} a$ kinase/nuclear factor- $\mathrm{kB}$, endoplasmic reticulum stressinduced unfolded protein response, and NOD-like receptor P3 - ultimately causing insulin intolerance and inflammation [34]. Although HFD-induced type 2 diabetes mellitus varies with the mouse strain used, duration of induction, and the treatment protocol, the murine model of HFD-induced obesity is still the best model for investigating the etiological, pathological, and treatment op- 
tions for this condition [35]. A previous study reported that mice fed the HFD for obesity induction can eventually develop physiological hyperglycemia and hyperlipidemia [36]. In the present study, we observed glucose intolerance and hyperlipidemia after inducing obesity in mice through an HFD for 9 weeks (Fig. 6; Table 3). Running on a treadmill can inhibit the endoplasmic reticulum stress associated with insulin resistance and hyperglycemia in HFD-induced obesity, possibly through regulation of the circulating myokine IL-15, which is secreted by skeletal muscle [37]. A previous study reported that (1) probiotics/prebiotics can exert glycemic control through the modulation of microbiota, including the production of short-chain fatty acids, secretion of incretin, and bile acid metabolism, and (2) probiotics can also be considered as a promising treatment strategy for obesity and diabetes [38]. We observed that compared with exercise or probiotics alone, a combination of exercise and OLP01 probiotics more greatly improved glucose tolerance.

The complications of obesity, such as hyperglycemia and hyperlipidemia, were also indicated by the clinical variables at the end of this study. An epidemiological cross-sectional study on patients with diabetes and obesity reported that serum UA level was significantly associated with obesity and BMI [39] and positively correlated with metabolic syndrome [40]. Moreover, individuals with obesity can also develop chronic kidney disease due to insulin resistance and hypertension, along with systemic inflammation and dyslipidemia. The therapeutic strategies for obesity include exercise, decrease of inflammation and oxidative stress, lowering of UA levels, and regulation of mineralocorticoid receptor signaling, which can improve tubulointerstitial fibrosis and further inhibit the progression of kidney disease in individuals with obesity and diabetes [41]. An international cohort study also reported that BUN level is positively associated with increased risk of diabetes mellitus and can be considered a useful predictor of adverse outcomes in patients with overweight or obesity [42]. The levels of glucose, BUN, CREA, UA, and lipid-related molecules were significantly elevated because of the HFD in the current model. Although the combined use of exercise training and OLP-01 probiotics significantly decreased these indexes when applied individually, a combination of exercise and OLP-01 probiotics more effectively improved these indexes, which improved health status. This combination may ameliorate the risk of associated comorbidities such as diabetes and chronic kidney disease associated with obesity; thus, it can be used as an alternative management strategy for obesity.

Exercise and Probiotics for Diet-Induced Obesity
B. longum may exist in varying abundance and population diversity in people with different ages, lifestyles, and eating habits, and the subspecies of B. longum isolated from nonathlete populations may have some of the microbial physiological activities of the B. longum OLP-1 strain. A previous study reported that 2 strain subspecies, infantis and longum, isolated from the infant and adult intestine had anti-inflammatory effects. However, the in vivo functional activities of $B$. longum strains isolated from nonathlete and healthy populations require further validation regarding their synergistic improvement of obesity management and health. The prevalence and severity of obesity-associated complications should also be further observed, and the effects of long-term obesity induction with probiotics and exercise interventions should be also evaluated. In addition, the role of microbiota and metabolites, which are affected by exercise, probiotics, and their combination, in the underlying mechanisms and physiological modulations in animal obesity models or individuals with obesity should be ascertained. The gut-brain axis, gut-muscle axis, and hypothalamic-pituitary-adrenal axis may be crucial associated mechanisms that modulate physiological regulation, such as energy metabolism, inflammation, oxidative stress, metabolic processes, thermogenesis, and nutrient bioavailability, and that are affected by microbiota composition as well as exercise and probiotics intervention.

\section{Conclusion}

Obesity is an important health issue, and its prevalence is on the increase. Therefore, previous studies have focused on physical activity, energy balance, and nutritional strategies to manage obesity. We observed the deleterious effects of obesity in terms of physical activity, hyperlipidemia, hyperglycemia, fat composition, and peripheral fatigue indexes, obtaining findings consistent with previously reported results. Although treadmill aerobic exercise in mice with HFD-induced obesity can improve weight management, glucose tolerance, and hyperlipidemia, the exercise-related physiological indexes of the HFD + EX group were nonsignificantly different to those of the HFD group. Furthermore, exercise of optimal intensity, along with sufficient rest, is critical to healthy weight loss in individuals with obesity, and OLP-01 probiotics can supplement regular exercise to aid obesity control and physiological adaptation.

Obes Facts 2021;14:306-319 317 


\section{Acknowledgement}

The authors are grateful to the graduate students, Yi-Ting Hsiao and Hsuan-Chen Liu from National Taipei University of Nursing and Health Sciences and National Taiwan Sport University for their technical assistance in animal management and experimental analysis. The manuscript was edited by Wallace Academic Editing.

\section{Statement of Ethics}

All animal experimental procedures were reviewed by the Institutional Animal Care and Use Committee (IACUC) of National Taiwan Sport University, and the guidelines of protocol IACUC-10801 were approved by the Ethics Committee of the IACUC.

\section{Funding Sources}

This study was supported by Taiwan's Ministry of Science and Technology (grant No.: MOST 109-2410-H-227 -006 -MY2 and MOST 108-2410-H-227-007).

\section{Author Contributions}

W.-C.H. designed the experiments; Y.-J.H. and M.-C.L. performed the laboratory experiments; C.-C.C. contributed the reagents, materials, pathological evaluations, and analysis platforms; Y.-J.H. and C.-C.C. analyzed the data and prepared the Figures and tables; and W.-C.H. interpreted the results, wrote the manuscript, and revised the manuscript.

\section{Conflict of Interest Statement}

The authors declare no conflicts of interest.

\section{References}

1 Abdelaal M, le Roux CW, Docherty NG. Morbidity and mortality associated with obesity. Ann Transl Med. 2017 Apr;5(7):161

2 Obesity: preventing and managing the global epidemic. Report of a WHO consultation. World Health Organ Tech Rep Ser. 2000; 894:i-xii.

3 Chang HC, Yang HC, Chang HY, Yeh CJ, Chen HH, Huang KC, et al. Morbid obesity in Taiwan: Prevalence, trends, associated social demographics, and lifestyle factors. PLoS One. 2017 Feb;12(2):e0169577.

4 Hruby A, Manson JE, Qi L, Malik VS, Rimm EB, Sun Q, et al. Determinants and Consequences of Obesity. Am J Public Health. 2016 Sep;106(9):1656-62.

5 Wang CY, Liao JK. A mouse model of dietinduced obesity and insulin resistance. Methods Mol Biol. 2012;821:421-33.

6 Donnelly JE, Honas JJ, Smith BK, Mayo MS, Gibson CA, Sullivan DK, et al. Aerobic exercise alone results in clinically significant weight loss for men and women: midwest exercise trial 2. Obesity (Silver Spring). 2013 Mar;21(3):E219-28.

7 Willis LH, Slentz CA, Bateman LA, Shields AT, Piner LW, Bales CW, et al. Effects of aerobic and/or resistance training on body mass and fat mass in overweight or obese adults. J Appl Physiol (1985). 2012 Dec;113(12):18317.

8 Boutcher SH. High-intensity intermittent exercise and fat loss. J Obes. 2011;2011:868305.

9 Cox CE. Role of Physical Activity for Weight Loss and Weight Maintenance. Diabetes Spectr. 2017 Aug;30(3):157-60.
10 Obert J, Pearlman M, Obert L, Chapin S. Popular Weight Loss Strategies: a Review of Four Weight Loss Techniques. Curr Gastroenterol Rep. 2017 Nov;19(12):61.

11 Paoli A. Ketogenic diet for obesity: friend or foe? Int J Environ Res Public Health. 2014 Feb;11(2):2092-107.

12 Syngai GG, Gopi R, Bharali R, Dey S, Lakshmanan GM, Ahmed G. Probiotics - the versatile functional food ingredients. J Food Sci Technol. 2016 Feb;53(2):921-33.

13 Anandharaj M, Sivasankari B, Rani RP. Effects of probiotics, prebiotics, and synbiotics on hypercholesterolemia: a review. Zhongguo Shengwuzhipinxue Zazhi. 2014;2014(Feb):1-7.

14 Schott EM, Farnsworth CW, Grier A, Lillis JA, Soniwala S, Dadourian GH, et al. Targeting the gut microbiome to treat the osteoarthritis of obesity. JCI Insight. 2018 Apr;3 (8):e95997.

15 In Kim H, Kim JK, Kim JY, Jang SE, Han MJ, Kim DH. Lactobacillus plantarum LC27 and Bifidobacterium longum LC67 simultaneously alleviate high-fat diet-induced colitis, endotoxemia, liver steatosis, and obesity in mice. Nutr Res. 2019 Jul;67:78-89.

16 Pinto-Sanchez MI, Hall GB, Ghajar K, Nardelli A, Bolino C, Lau JT, et al. Probiotic Bifidobacterium longum NCC3001 Reduces Depression Scores and Alters Brain Activity: A Pilot Study in Patients With Irritable Bowel Syndrome. Gastroenterology. 2017 Aug;153 (2):448-59.e8.
17 Odamaki T, Bottacini F, Kato K, Mitsuyama E, Yoshida K, Horigome A, et al. Genomic diversity and distribution of Bifidobacterium longum subsp. longum across the human lifespan. Sci Rep. 2018 Jan;8(1):85.

18 Fontané L, Benaiges D, Goday A, Llauradó G, Pedro-Botet J. Influence of the microbiota and probiotics in obesity. Clin Investig Arterioscler. 2018 Nov - Dec;30(6):271-9.

19 Huang WC, Wei CC, Huang CC, Chen WL, Huang HY. The Beneficial Effects of Lactobacillus plantarum PS128 on High-Intensity, Exercise-Induced Oxidative Stress, Inflammation, and Performance in Triathletes. Nutrients. 2019 Feb;11(2):353.

20 Huang WC, Hsu YJ, Huang CC, Liu HC, Lee MC. Exercise Training Combined with Bifidobacterium longum OLP-01 Supplementation Improves Exercise Physiological Adaptation and Performance. Nutrients. 2020 Apr;12(4):1145.

21 Foster-Schubert KE, Alfano CM, Duggan CR, Xiao L, Campbell KL, Kong A, et al. Effect of diet and exercise, alone or combined, on weight and body composition in overweight-to-obese postmenopausal women. Obesity (SilverSpring).2012 Aug;20(8):162838.

22 Huang WC, Chang WC, Hsu YJ, Huang CF, Huang CC, Kao CY, et al. The modulative effects of microcurrent electrical nerve stimulation on diabetic mice. Chin J Physiol. 2017 Feb;60(1):62-72. 
23 Alahmari KA, Silvian SP, Reddy RS, Kakaraparthi VN, Ahmad I, Alam MM. Hand grip strength determination for healthy males in Saudi Arabia: A study of the relationship with age, body mass index, hand length and forearm circumference using a hand-held dynamometer. J Int Med Res. 2017 Apr;45(2):540-8.

24 Kim DH, Kim SH, Jeong WS, Lee HY. Effect of BCAA intake during endurance exercises on fatigue substances, muscle damage substances, and energy metabolism substances. J Exerc Nutrition Biochem. 2013 Dec;17(4):169-80.

25 Kim B, Kwon J, Kim MS, Park H, Ji Y, Holzapfel W, et al. Protective effects of Bacillus probiotics against high-fat diet-induced metabolic disorders in mice. PLoS One. 2018 Dec;13(12):e0210120.

26 Youssef MK. The impact of obesity on walking and physical performance. Egypt J Intern Med. 2014 Jan;26(2):40-4.

27 Nantel J, Mathieu ME, Prince F. Physical activity and obesity: biomechanical and physiological key concepts. J Obes. 2011;2011:650230.

28 Stenholm S, Sallinen J, Koster A, Rantanen T, Sainio P, Heliövaara M, et al. Association between obesity history and hand grip strength in older adults-exploring the roles of inflammation and insulin resistance as mediating factors. J Gerontol A Biol Sci Med Sci. 2011 Mar;66(3):341-8
29 Huang CC, Tseng TL, Huang WC, Chung $\mathrm{YH}$, Chuang HL, Wu JH. Whole-body vibration training effect on physical performance and obesity in mice. Int J Med Sci. 2014 Sep;11(12):1218-27.

30 Isik O, Dogan I. Effects of bilateral or unilateral lower body resistance exercises on markers of skeletal muscle damage. Biomed J. 2018 Dec;41(6):364-8.

31 Chen CJ, Liao YH, Lin SY, Yu JX, Li ZJ, Lin YC, et al. Diet-induced obesity accelerates blood lactate accumulation of rats in response to incremental exercise to maximum. Am J Physiol Regul Integr Comp Physiol. 2017 Nov;313(5):R601-7.

32 Vincent HK, Morgan JW, Vincent KR. Obesity exacerbates oxidative stress levels after acute exercise. Med Sci Sports Exerc. 2004 May;36(5):772-9.

33 Cho SY, Roh HT. Trekking poles reduce downhill walking-induced muscle and cartilage damage in obese women. J Phys Ther Sci. 2016 May;28(5):1574-6.

34 Ringseis R, Eder K, Mooren FC, Krüger K. Metabolic signals and innate immune activation in obesity and exercise. Exerc Immunol Rev. 2015;21:58-68.

35 Heydemann A. An Overview of Murine High Fat Diet as a Model for Type 2 Diabetes Mellitus. J Diabetes Res. 2016;2016:2902351.
36 Park SH, Ko SK, Choi JG, Chung SH. Salicornia herbacea prevents high fat diet-induced hyperglycemia and hyperlipidemia in ICR mice. Arch Pharm Res. 2006 Mar;29(3):25664.

37 Yang HT, Luo LJ, Chen WJ, Zhao L, Tang CS, Qi YF, et al. IL-15 expression increased in response to treadmill running and inhibited endoplasmic reticulum stress in skeletal muscle in rats. Endocrine. 2015 Feb;48(1):152-63.

38 Gérard C, Vidal H. Impact of Gut Microbiota on Host Glycemic Control. Front Endocrinol (Lausanne). 2019 Jan;10:29.

39 Ali N, Perveen R, Rahman S, Mahmood S, Rahman S, Islam S, et al. Prevalence of hyperuricemia and the relationship between serum uric acid and obesity: A study on Bangladeshi adults. PLoS One. 2018 Nov;13(11):e0206850.

40 Huang G, Xu J, Zhang T, Cai L, Liu H, Yu X, et al. Hyperuricemia is associated with metabolic syndrome in the community very elderly in Chengdu. Sci Rep. 2020 May;10(1):8678.

41 Whaley-Connell A, Sowers JR. Obesity and kidney disease: from population to basic science and the search for new therapeutic targets. Kidney Int. 2017 Aug;92(2):313-23.

42 Iwasaki Y, Takata Y, Itou Y, Yamaguchi T, Watanabe M, Chikamori T. Blood Urea Nitrogen Is a Predictor of Adverse Outcomes in Overweight or Obese Patients with Acute Decompensated Heart Failure. J Card Fail. 2017 Oct;23(10):S44. 